\title{
Calcified Pericarditis associated with Ventricular Septal Defect: Should We Close the Hole or Open Our Eyes?
}

\section{Ferroni F, Bordese R, Veglia S and Agnoletti G*}

Division of Paediatric Cardiology and Radiology, Città della Salute e della Scienza, Turin, Italy

\begin{abstract}
We present the case of a 45-year-old woman with recent onset of dipnea and atrial fibrillation. She was referred to our center for treatment of an associated ventricular septal defect. Echocardiography confirmed the presence of a membranous ventricular septal defect, but also showed signs of constriction. Cardiac computed tomography displayed a severe, diffuse calcified pericarditis. We show that, in adult patients, a minor congenital heart disease can catalyze the clinician's attention and, like in this case, mask major signs of acquired heart disease.
\end{abstract}

Keywords: Constrictive pericarditis; Computed tomography; Ventricular septal defect

\section{Introduction}

Constrictive pericarditis is a rare entity that has multiple possible causes and is associated with variable clinical findings, depending on its severity. It develops insidiously, and in many cases, no aetiology is ever determined [1]. A diagnosis solely on the basis of the clinical history is virtually impossible. Dyspnea is the most common presenting symptom and occurs in virtually all patients. Definitive diagnosis is made with non-invasive imaging [2]. Treatment is surgical. Complete recovery is common, mostly in idiopathic forms [3]. We present the case of a young woman whose medical history started with the recent onset of atrial fibrillation. She was referred to our center to evaluate and treat an associated congenital heart disease.

\section{Case Report}

A 45 year-old woman working in the cleaning industry, was free of symptoms until a few months before, when she started to complain about palpitations and dyspnea. ECG showed high frequency atrial fibrillation. Echogardiography highlighted bilateral atrial enlargement, and a membranous ventricular septal defect (VSD). The patient received three electrical shocks that only transiently restored sinus rhythm. She was treated with anticoagulants, diuretics, and beta-blockers, then she was referred to our center for evaluation and treatment of her VSD. At clinical examination, she was in good general condition, had a meso-systolic murmur, and a normal second sound. We noticed jugular venous distention. At abdominal palpation the liver was mildly enlarged with a solid margin. Trans-thoracic echocardiography was performed. We confirmed the presence of a small patency in a large aneurysm of the ventricular septum (Figure 1A). Both ventricles had a preserved systolic function (Video 1). Inferior vena cava was dilated $(27 \mathrm{~mm})$ and not collapsing. The estimated gradient through the VSD was $80 \mathrm{mmHg}$ and the velocity of a moderate tricuspid regurgitation $2 \mathrm{~m} / \mathrm{sec}$, suggesting low pulmonary pressure. ECG confirmed atrial fibrillation (Figure 1B). We noticed a round hyperechogenic formation that seemed to compress the right ventricle, measuring $3 \times 5 \mathrm{~cm}$ (Video 1). The presence of atrial fibrillation did not allow to perform cardiac MRI, thus the patient underwent a 64-row multidetector computed tomography. It showed a severely calcified pericardium and a round mass, likely corresponding to pericardial fat, in front of the right ventricle (Figures 1C and 1D). The liver was enlarged, with lobulated contour and inhomogeneous parenchyma, suggesting longlasting venous stasis. No focal lesions evoking previous tuberculosis were found in the lungs. At cardiac catheterization mean atrial and ventricular end-diastolic pressures were $15 \mathrm{mmHg}$. Pulmonary arterial pressure was $25 / 16 / 20 \mathrm{mmHg}$. Diastolic filling showed a typical square root sign. Coronary arteries were normal.

The patient underwent pericardiectomy and concomitant closure of the ventricular septal defect. At surgical examination calcific infiltration interested both parietal and visceral pericardium (Figure 2A). The surgeon removed a $4 \mathrm{~cm}$ thick calcified mass deeply infiltrating the atrio-ventricular groove and tightly attached to the right ventricular wall (Figure 2B). Postoperative period was uneventful. Postoperative echocardiopgraphy showed normal volume and filling of both ventricles, preserved systolic function of the left ventricle, mildly depressed systolic function of the right ventricle. The patient was discharged in atrial fibrillation after 7 days. She continued to be treated with anticoagulants and beta-blockers.

\section{Discussion}

Calcified constrictive pericarditis is a rare entity, typically idiopathic $[1,2]$ or due to tuberculosis $[4,5]$. It is a potentially curable disease if diagnosed early, but it is potentially fatal if overlooked. This can happen because symptoms may develop slowly over a number of years. Finally, systolic ventricular dysfunction can occur. The initial history may be more compatible with liver disease, because of the predominance of findings related to the venous system. Several authors have shown that, like in our patient, the liver can be severely affected by chronic venous stasis [6].

In symptomatic patients the presence of small ventricles, enlarged atria, diastolic restrictive Doppler pattern associated with enlarged liver, should raise the suspicion of constrictive or restrictive diseases. In our patient the presence of a round mass in front of the right ventricle should have supported the correct diagnosis. However, CT scanning

*Corresponding author: Gabriella Agnoletti, Division of Paediatric Cardiology and Radiology, Città della Salute e della Scienza, Turin, Italy, Tel: 0039-011-3135541; Fax: 0039-011-3135482; E-mail: gagnoletti@cittadellasalute.to.it

Received May 11, 2016; Accepted May 17, 2016; Published May 23, 2016

Citation: Ferroni F, Bordese R, Veglia S, Agnoletti G (2016) Calcified Pericarditis associated with Ventricular Septal Defect: Should We Close the Hole or Open Our Eyes? J Cardiovasc Dis Diagn S1: 004. doi:10.4172/2329-9517.S1-004

Copyright: @ 2016 Ferroni F, et al. This is an open-access article distributed under the terms of the Creative Commons Attribution License, which permits unrestricted use, distribution, and reproduction in any medium, provided the original author and source are credited. 


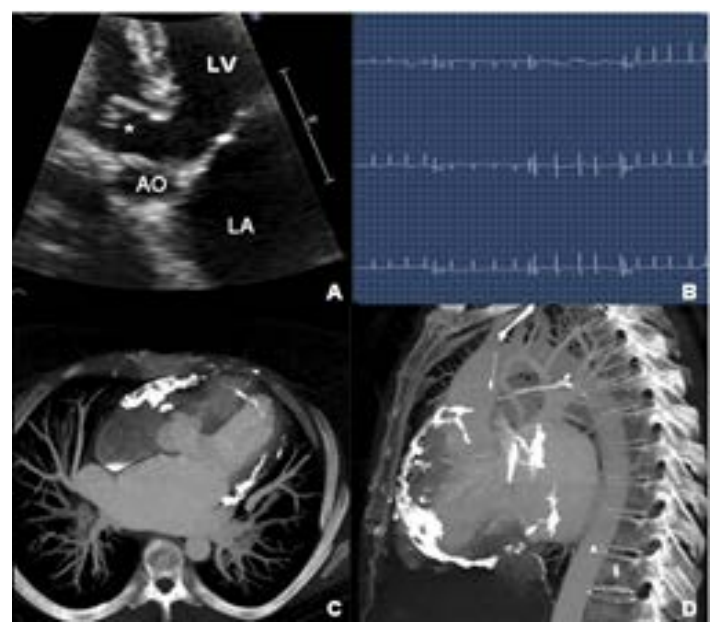

Figure 1: A, B, C and D) At trans-thoracic echocardiography in 5-chamber view an aneurysm of the membranous ventricular septum is noticed $\left(^{*}\right)$. A patency can be seen close to the aortic valve. LA: left atrium, LV: left ventricle, AO: aorta (1A).

ECG shows rapid atrial fibrillation (120 bpm) (1B).

In 5 chamber view cardiac tomography shows a severely calcified pericardium and a thick mass, likely corresponding to pericardial fat, in correspondence of the atrio-ventricular groove. Ventricles are small. The aneurysm of the membranous septum is clearly seen. Both atria are enlarged (1C).

In sagittal view the entire heart appears wrapped by calcified pericardium. Calcium infiltration also involves the atrio-ventricular junction (1D).

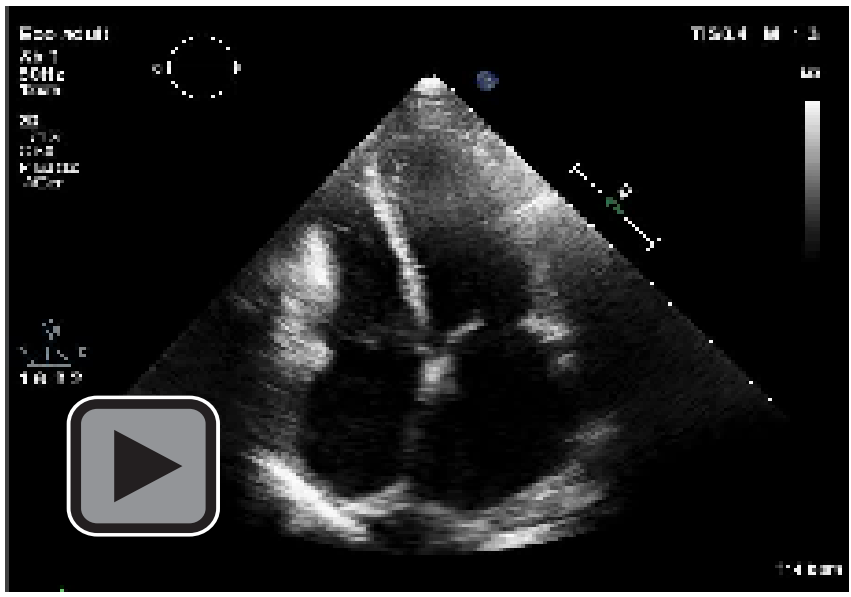

Video 1: Round hyperechogenic formation that seemed to compress the right ventricle, measuring $3 \times 5 \mathrm{~cm}$.

and MRI are considered the procedures of choice for imaging the pericardium [2].

Although the association of constrictive pericarditis with atrial fibrillation is not common, it has been reported that the presence of pericardial calcification and the increasing disease duration are associated with a higher chance of developing this arrhythmia [7].

The long-term outcome of operated patients has been shown to be more favourable in younger patients without co-morbidities. The best outcome is observed in idiopathic forms, while the degree of pericardial calcification has shown no effect on survival [3]. Although in our patient the surgeon found a deep infiltration in the right ventricular wall, postoperative echocardiography showed an almost normal right ventricular function and a small inferior vena cava. Thus, we hope that

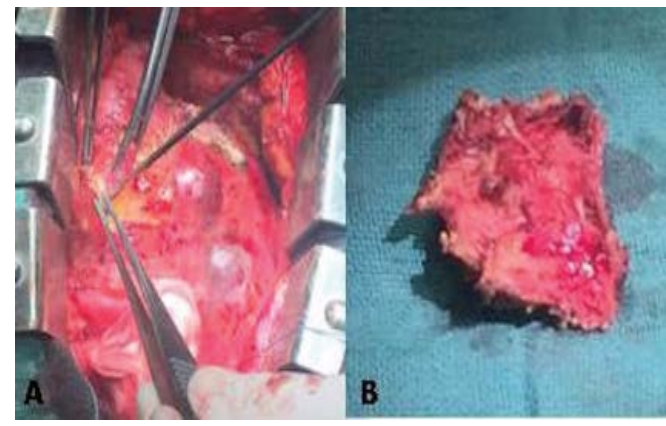

Figure 2: A and B) Intra-operative findings. At surgical inspection the visceral pericardium is whitish. The surgeon is removing a thick mass infiltrating the atrio-ventricular groove (A). The mass measures $4 \times 5 \times 2 \mathrm{~cm}$ and is hard, likely constituted by calcium and fibrous tissue (B).

the hepatic congestion will, at least partially, regress. Our patient was symptom - free and active until a few months before. We suppose that palpitations and dyspnea were the consequence of the recent onset of atrial fibrillation. Has it can occur in these patients the progression of the disease was slow and only a concomitant finding lead to the correct diagnosis. In this particular case our findings put forward for consideration that in adult patients a minor congenital heart disease can catalyze the clinician's attention and, like in this case, mask major signs of acquired heart disease.

\section{References}

1. Peebles CR, Shambrook JS, Harden SP (2011) Pericardial disease--anatomy and function. Br J Radiol 84 Spec No 3: S324-337.

2. Bogaert J, Meyns B, Dymarkowski S, Sinnaeve P, Meuris B (2015) Calcified Constrictive Constrictive Pericarditis: Prevalence, Distribution Patterns, and Relationship to the Myocardium. JACC Cardiovasc Imaging.

3. Bertog SC, Thambidorai SK, Parakh K, Schoenhagen P, Ozduran V, et al. (2004) Constrictive pericarditis: etiology and cause-specific survival after pericardiectomy. J Am Coll Cardiol 43: 1445-1452.

4. Osada H, Nakajima H (2014) Porcelain heart: severely calcified pericardium due to Mycobacterium tuberculosis. Asian Cardiovasc Thorac Ann 22: 229.

5. Sanhueza ME, Torres R, Segura P, Villalobos A, Segovia E (2016) The Fortress Becomes a Prison: Calcified Constrictive Pericarditis. Am J Med 129: 263-265.

6. Lominadze Z, Kia L, Shah S, Parekh K, Levitsky J (2015) Constrictive Pericarditis as a Cause of Refractory Ascites. ACG Case Rep J 2: 175-177.

7. Rezaian GR, Poor-Moghaddas M, Kojuri J, Rezaian S, Liaghat L, et al. (2009) Atrial fibrillation in patients with constrictive pericarditis: the significance of pericardial calcification. Ann Noninvasive Electrocardiol 14: 258-261. 